Article

\title{
A Parametric Kind of Fubini Polynomials of a Complex Variable
}

\author{
Sunil Kumar Sharma ${ }^{1, * \mathbb{D}}$, Waseem A. Khan ${ }^{2}$ and Cheon Seoung Ryoo ${ }^{3}$ \\ 1 College of Computer and Information Sciences, Majmaah University, Majmaah 11952, Saudi Arabia \\ 2 Department of Mathematics and Natural Sciences, Prince Mohammad Bin Fahd University, P.O. Box 1664, \\ Al Khobar 31952, Saudi Arabia; wkhan1@pmu.edu.sa \\ 3 Department of Mathematics, Hannam University, Daejeon 34430, Korea; ryoocs@hnu.kr \\ * Correspondence: s.sharma@mu.edu.sa
}

Received: 31 March 2020; Accepted: 16 April 2020; Published: 22 April 2020

check for updates

\begin{abstract}
In this paper, we propose a parametric kind of Fubini polynomials by defining the two specific generating functions. We also investigate some analytical properties (for example, summation formulae, differential formulae and relationships with other well-known polynomials and numbers) for our introduced polynomials in a systematic way. Furthermore, we consider some relationships for parametric kind of Fubini polynomials associated with Bernoulli, Euler, and Genocchi polynomials and Stirling numbers of the second kind.
\end{abstract}

Keywords: Bernoulli polynomials; Euler polynomials; Genocchi polynomials; Fubini polynomials; Stirling numbers

MSC: 11B 68; 11B73; 11C08; 11Y35

\section{Introduction}

Mathematicians and other scientists have studied trigonometric functions, special numbers, and polynomials, and their applications because these functions have various mathematical usages which include derivative, integral and other algebraic properties. By using these functions with their functional equations and derivative equations, various properties of these special numbers and polynomials have been investigated (see [1-26]). By using these functions with a trigonometric function, we not only study some special families of polynomials and numbers including the Bernoulli, Euler, and Genocchi polynomials, but also derive some identities and relationships for these polynomials and numbers.

The classical Bernoulli polynomials $B_{j}(u)$, the classical Euler polynomials $E_{j}(u)$ and the classical Genocchi polynomials $G_{j}(u)$ are usually defined by means of the following generating functions

$$
\begin{aligned}
& \left(\frac{z}{e^{z}-1}\right) e^{u z}=\sum_{j=0}^{\infty} B_{j}(u) \frac{z^{j}}{j !}, \quad(|z|<2 \pi) \\
& \left(\frac{2}{e^{z}+1}\right) e^{u z}=\sum_{j=0}^{\infty} E_{j}(u) \frac{z^{j}}{j !}, \quad(|z|<\pi)
\end{aligned}
$$

and

$$
\left(\frac{2 z}{e^{z}+1}\right) e^{u z}=\sum_{j=0}^{\infty} G_{j}(u) \frac{z^{j}}{j !}, \quad \quad(|z|<\pi)
$$

respectively. Each of these polynomials has been extensively studied in many recent works, (see $[18,19])$. 
The Geometric (also known as Fubini) polynomials [1] are defined by

$$
\frac{1}{1-u\left(e^{z}-1\right)}=\sum_{j=0}^{\infty} F_{j}(u) \frac{z^{j}}{j !}
$$

so that

$$
F_{j}(u)=\sum_{k=0}^{j} k ! S_{2}(j, k) u^{k}=\sum_{k=0}^{j}\left\{\begin{array}{l}
j \\
k
\end{array}\right\} k ! u^{k}
$$

where $\left\{\begin{array}{l}j \\ k\end{array}\right\}$ are called the Stirling numbers of second kind, (see [13,17]).

On setting $u=1$ in (4), we obtain

$$
\frac{1}{2-e^{z}}=\sum_{j=0}^{\infty} F_{j} \frac{z^{j}}{j !}
$$

where $F_{j}$ are called the $j$ th Fubini numbers or ordered Bell numbers, (see $\left.[4,26]\right)$.

A few numbers of these polynomials are

$$
\begin{gathered}
F_{0}(u)=1, F_{1}(u)=u, F_{2}(u)=u+2 u^{2}, \\
F_{3}(u)=u+6 u^{2}+6 u^{3}, F_{4}(u)=u+14 u^{2}+36 u^{3}+24 u^{4},
\end{gathered}
$$

and

$$
F_{0}=1, F_{1}=1, F_{2}=3, F_{3}=13, F_{4}=75 .
$$

The Stirling numbers of the first kind are defined by the coefficients in the expansion of $(u)_{j}$ in terms of powers of $u$ as follows, (see [14])

$$
(u)_{j}=u(u-1) \cdots(u-j+1)=\sum_{l=0}^{j} s_{1}(j, l) u^{l}, \quad(j \geq 0),
$$

and the Stirling numbers of the second kind are defined by (see $[15,16])$

$$
\left(e^{z}-1\right)^{j}=j ! \sum_{l=j}^{\infty} S_{2}(l, j) \frac{z^{l}}{l !},(j \geq 0)
$$

Recently, Masjed-Jamei et al. [6-9] and Srivastava et al. [23-25] introduced and studied the parametric kind of the two exponential generating functions $e^{u z} \cos v z$ and $e^{u z} \sin v z$ are defined by

$$
e^{u z} \cos v z=\sum_{k=0}^{\infty} C_{k}(u, v) \frac{z^{k}}{k !}
$$

and

$$
e^{u z} \sin v z=\sum_{k=0}^{\infty} S_{k}(u, v) \frac{z^{k}}{k !}
$$

where

$$
C_{k}(u, v)=\sum_{j=0}^{\left[\frac{k}{2}\right]}(-1)^{j}\left(\begin{array}{l}
k \\
2 j
\end{array}\right) u^{k-2 j} v^{2 j},
$$


and

$$
S_{k}(u, v)=\sum_{j=0}^{\left[\frac{k-1}{2}\right]}(-1)^{j}\left(\begin{array}{l}
k \\
2 j+1
\end{array}\right) u^{k-2 j-1} v^{2 j+1} .
$$

In (2018), Kim and Ryoo [11] introduced the cosine-Bernoulli polynomials of a complex variable, the sine-Bernoulli polynomials of a complex variable and the cosine-Euler polynomials of a complex variable, the sine-Euler polynomials of a complex variable, respectively are defined as follows

$$
\frac{z}{e^{z}-1} e^{(u+i v) z}=\sum_{j=0}^{\infty} B_{j}(u+i v) \frac{z^{j}}{j !}
$$

and

$$
\frac{2}{e^{z}+1} e^{(u+i v) z}=\sum_{j=0}^{\infty} E_{j}(u+i v) \frac{z^{j}}{j !} .
$$

From (13) and (14), we get

$$
\frac{z}{e^{z}-1} e^{u z} \cos v z=\sum_{j=0}^{\infty} \frac{B_{j}(u+i v)+B_{j}(u-i v)}{2} \frac{z^{j}}{j !}=\sum_{j=0}^{\infty} B_{j}^{(c)}(u, v) \frac{z^{j}}{j !},
$$

and

$$
\begin{aligned}
& \frac{2}{e^{z}-1} e^{u z} \sin v z=\sum_{j=0}^{\infty} \frac{B_{j}(u+i v)-B_{j}(u-i v)}{2 i} \frac{z^{j}}{j !}=\sum_{j=0}^{\infty} B_{j}^{(s)}(u, v) \frac{z^{j}}{j !}, \\
& \frac{2}{e^{z}+1} e^{u z} \cos v z=\sum_{j=0}^{\infty} \frac{E_{j}(u+i v)+E_{j}(u-i v)}{2} \frac{z^{j}}{j !}=\sum_{j=0}^{\infty} E_{j}^{(c)}(u, v) \frac{z^{j}}{j !},
\end{aligned}
$$

and

$$
\frac{2}{e^{z}+1} e^{u z} \sin v z=\sum_{j=0}^{\infty} \frac{E_{j}(u+i v)-E_{j}(u-i v)}{2 i} \frac{z^{j}}{j !}=\sum_{j=0}^{\infty} E_{j}^{(s)}(u, v) \frac{z^{j}}{j !} .
$$

The main object of this paper is as follows. In Section 2, we consider generating a function for the parametric type of Fubini numbers and polynomials of a complex variable and give some basic properties of these polynomials. In Section 3, we derive recurrence relations, differentiation, summation formulae of parametric Fubini-type polynomials. In Section 4, we construct relationships for parametric Fubini-type polynomials associated with Bernoulli, Euler, Genocchi polynomials and Stirling numbers of the second kind.

\section{Two Parametric Kind of the Fubini Polynomials of Complex Variable}

In this section, we introduce the cosine-Fubini polynomials and sine-Fubini polynomials by splitting complex Fubini polynomials into real $\Re$ and imaginary $\Im$ parts and present some basic properties. Now, we consider the Fubini polynomials that are given by the generating function

$$
\frac{1}{1-w\left(e^{z}-1\right)} e^{(u+i v) z}=\sum_{j=0}^{\infty} F_{j}(u+i v ; w) \frac{z^{j}}{j !} .
$$

The well-known Euler's formula is defined as follows (see [11])

$$
e^{(u+i v) z}=e^{u z} e^{i v z}=e^{u z}(\cos v z+i \sin v z) .
$$

Using (15) and (16), we have

$$
\sum_{j=0}^{\infty} F_{j}(u+i v ; w) \frac{z^{j}}{j !}=\frac{1}{1-w\left(e^{z}-1\right)} e^{(u+i v) z}=\frac{1}{1-w\left(e^{z}-1\right)} e^{u z}(\cos v z+i \sin v z),
$$


and

$$
\sum_{j=0}^{\infty} F_{j}(u-i v ; w) \frac{z^{j}}{j !}=\frac{1}{1-w\left(e^{z}-1\right)} e^{(u-i v) z}=\frac{1}{1-w\left(e^{z}-1\right)} e^{u z}(\cos v z-i \sin v z) .
$$

From (17) and (18), we get

$$
\frac{1}{1-w\left(e^{z}-1\right)} e^{u z} \cos v z=\sum_{j=0}^{\infty}\left(\frac{F_{j}(u+i v ; w)+F_{j}(u-i v ; w)}{2}\right) \frac{z^{j}}{j !},
$$

and

$$
\frac{1}{1-w\left(e^{z}-1\right)} e^{u z} \sin v z=\sum_{j=0}^{\infty}\left(\frac{F_{j}(u+i v ; w)-F_{j}(u-i v ; w)}{2 i}\right) \frac{z^{j}}{j !}
$$

Definition 1. Two parametric kinds of Fubini polynomials or the cosine-Fubini polynomials $F_{j}^{(c)}(u, v ; w)$ and sine-Fubini polynomials $F_{j}^{(s)}(u, v ; w)$ for nonnegative integer $j$ are defined by

$$
\frac{1}{1-w\left(e^{z}-1\right)} e^{u z} \cos v z=\sum_{j=0}^{\infty} F_{j}^{(c)}(u, v ; w) \frac{z^{j}}{j !}
$$

and

$$
\frac{1}{1-w\left(e^{z}-1\right)} e^{u z} \sin v z=\sum_{j=0}^{\infty} F_{j}^{(s)}(u, v ; w) \frac{z^{j}}{j !}
$$

respectively.

It is clear that

$$
\begin{gathered}
F_{n}^{(c)}(u, 0 ; w)=F_{n}^{(c)}(u ; w), F_{n}^{(s)}(u, 0 ; w)=0, F_{n}^{(c)}(0, v ; w)=F_{n}^{(c)}(v ; w), \\
F_{n}^{(s)}(0, v ; w)=F_{n}^{(s)}(v ; w), F_{n}^{(c)}(0,0 ; 1)=F_{n}^{(c)}, F_{n}^{(s)}(0,0 ; w)=0 .
\end{gathered}
$$

The first few follow immediately from this generating function:

$$
\begin{aligned}
F_{0}^{(c)}(u, v ; w)= & 1 \\
F_{1}^{(c)}(u, v ; w)= & u+w, \\
F_{2}^{(c)}(u, v ; w)= & u^{2}-v^{2}+w+2 u w+2 w^{2} \\
F_{3}^{(c)}(u, v ; w)= & u^{3}-3 u v^{2}+w+3 u w+3 u^{2} w-3 v^{2} w+6 w^{2}+6 u w^{2}+6 z^{3}, \\
F_{4}^{(c)}(u, v ; w)= & u^{4}-6 u^{2} v^{2}+v^{4}+w+4 u w+6 u^{2} w+4 u^{3} w-6 v^{2} w-12 u v^{2} w+14 w^{2}+24 u w^{2} \\
& +12 u^{2} w^{2}-12 v^{2} w^{2}+36 w^{3}+24 u w^{3}+24 w^{4},
\end{aligned}
$$

and

$$
\begin{aligned}
& F_{0}^{(s)}(u, v ; w)=0 \\
& F_{1}^{(s)}(u, v ; w)=v \\
& F_{2}^{(s)}(u, v ; w)=2 u v+2 v w \\
& F_{3}^{(s)}(u, v ; w)=3 u^{2} v-v^{3}+3 v w+6 u v w+6 v w^{2}, \\
& F_{4}^{(s)}(u, v ; w)=4 u^{3} v-4 u v^{3}+4 v w+12 u v w+12 u^{2} v w-4 v^{3} w+24 v w^{2}+24 u v w^{2}+24 v w^{3} .
\end{aligned}
$$


From (19)-(22), we find

$$
\begin{aligned}
& F_{j}^{(c)}(u, v ; w)=\frac{F_{j}(u+i v ; w)+F_{j}(u-i v ; w)}{2}, \\
& F_{j}^{(s)}(u, v ; w)=\frac{F_{j}(u+i v ; w)-F_{j}(u-i v ; w)}{2 i} .
\end{aligned}
$$

Remark 1. Taking $u=0$ in (21) and (22), we get new type of polynomials as follows

$$
\frac{1}{1-w\left(e^{z}-1\right)} \cos v z=\sum_{j=0}^{\infty} F_{j}^{(c)}(0, v ; w) \frac{z^{j}}{j !}
$$

and

$$
\frac{1}{1-w\left(e^{z}-1\right)} \sin v z=\sum_{j=0}^{\infty} F_{j}^{(s)}(0, v ; w) \frac{z^{j}}{j !}
$$

respectively.

Remark 2. For $w=-\frac{1}{2}$ in (19) and (20), we have

$$
\frac{2}{e^{z}+1} e^{u z} \cos v z=\sum_{j=0}^{\infty}\left(\frac{E_{j}(u+i v)+E_{j}(u-i v)}{2}\right) \frac{z^{j}}{j !},
$$

and

$$
\frac{2}{e^{z}+1} e^{u z} \sin v z=\sum_{j=0}^{\infty}\left(\frac{E_{j}(u+i v)-E_{j}(u-i v)}{2 i}\right) \frac{z^{j}}{j !}
$$

(see [11]).

Remark 3. For $w=-\frac{1}{2}$ in (21) and (22), we get

$$
\sum_{j=0}^{\infty} F_{j}^{(c)}\left(u, v ;-\frac{1}{2}\right) \frac{z^{j}}{j !}=\frac{2}{e^{z}+1} e^{u z} \cos v z=\sum_{j=0}^{\infty} E_{j}^{(c)}(u, v) \frac{z^{j}}{j !},
$$

and

$$
\sum_{j=0}^{\infty} F_{j}^{(s)}\left(u, v ;-\frac{1}{2}\right) \frac{z^{j}}{j !}=\frac{2}{e^{z}+1} e^{u z} \sin v z=\sum_{j=0}^{\infty} E_{j}^{(s)}(u, v) \frac{z^{j}}{j !},
$$

(see [6]).

Now, we start some basic properties of these polynomials.

Theorem 1. Let $j \geq 0$, we have

$$
F_{j}^{(c)}(0, v ; w)=\sum_{r=0}^{\left[\frac{j}{2}\right]}\left(\begin{array}{l}
j \\
2 r
\end{array}\right)(-1)^{r} v^{2 r} F_{j-2 r}(w),
$$

and

$$
F_{j}^{(s)}(0, v ; w)=\sum_{r=0}^{\left[\frac{j-1}{2}\right]}\left(\begin{array}{l}
j \\
2 r+1
\end{array}\right)(-1)^{r} v^{2 r+1} F_{j-2 r-1}(w) .
$$


Proof. From (23) and (24), we have

$$
\begin{gathered}
\sum_{j=0}^{\infty} F_{j}^{(c)}(0, v ; w) \frac{z^{j}}{j !}=\frac{1}{1-w\left(e^{z}-1\right)} \cos v z \\
=\sum_{j=0}^{\infty} F_{j}(w) \frac{z^{j}}{j !} \sum_{r=0}^{\infty}(-1)^{r} v^{2 r} \frac{z^{2 r}}{(2 r) !} . \\
=\sum_{j=0}^{\infty}\left(\sum_{r=0}^{\left[\frac{j}{2}\right]}\left(\begin{array}{l}
j \\
2 r
\end{array}\right)(-1)^{r} v^{2 r} F_{j-2 r}(w)\right) \frac{z^{j}}{j !}
\end{gathered}
$$

and

$$
\begin{gathered}
\sum_{j=0}^{\infty} F_{j}^{(s)}(0, v ; w) \frac{z^{j}}{j !}=\frac{1}{1-w\left(e^{z}-1\right)} \sin v z \\
=\sum_{j=0}^{\infty}\left(\sum_{r=0}^{\left[\frac{j-1}{2}\right]}\left(\begin{array}{l}
j \\
2 r+1
\end{array}\right)(-1)^{r} v^{2 m+1} F_{j-2 r-1}(w)\right) \frac{z^{j}}{j !} .
\end{gathered}
$$

Therefore, by (27) and (28), we get the results (25) and (26).

Theorem 2. Let $j \geq 0$, we have

$$
\begin{gathered}
F_{j}(u+i v ; w)=\sum_{r=0}^{j}\left(\begin{array}{l}
j \\
r
\end{array}\right)(u+i v)^{j-r} F_{r}(w) \\
=\sum_{r=0}^{j}\left(\begin{array}{l}
j \\
r
\end{array}\right)(i v)^{j-r} F_{r}(u ; w)
\end{gathered}
$$

and

$$
\begin{gathered}
F_{j}(u-i v ; w)=\sum_{r=0}^{j}\left(\begin{array}{c}
j \\
r
\end{array}\right)(u-i v)^{j-r} F_{r}(w) \\
=\sum_{r=0}^{j}\left(\begin{array}{l}
j \\
r
\end{array}\right)(-1)^{j-r}(i v)^{j-r} F_{r}(u ; w) .
\end{gathered}
$$

Proof. By using (17) and (18), we can easily get. So we omit the proof.

Theorem 3. Let $j \geq 0$ and $v \neq 0$. Then

$$
F_{j}^{(c)}(u, v ; w)=H_{j}^{(c)}\left(u, v ; \frac{1+w}{w}\right)
$$

and

$$
F_{j}^{(s)}(u, v ; w)=H_{j}^{(s)}\left(u, v ; \frac{1+w}{w}\right),
$$

where $H_{j}(u)$ are called the Frobenius-Euler polynomials, (see [10,12]).

Proof. By (21), we have

$$
\begin{gathered}
\sum_{j=0}^{\infty} F_{j}^{(c)}(u, v ; w) \frac{z^{j}}{j !}=\frac{1}{1-w\left(e^{z}-1\right)} e^{u z} \cos v z \\
=\left(\frac{1-\frac{1+w}{w}}{e^{z}-\frac{1+w}{w}}\right) e^{u z} \cos v z
\end{gathered}
$$




$$
=\sum_{j=0}^{\infty} H_{j}^{(c)}\left(u, v ; \frac{1+w}{w}\right) \frac{z^{j}}{j !} .
$$

Comparing the coefficients of $\frac{t^{n}}{n !}$ on both sides, we obtain (31). The proof of (32) is similar.

Theorem 4. Let $j \geq 0$, we have

$$
\begin{aligned}
& F_{j}^{(c)}(u, v ; w)=\sum_{k=0}^{n}\left(\begin{array}{c}
j \\
k
\end{array}\right) F_{k}(w) C_{j-k}(u, v), \\
& F_{j}^{(s)}(u, v ; w)=\sum_{k=0}^{j}\left(\begin{array}{c}
j \\
k
\end{array}\right) F_{k}(w) S_{j-k}(u, v) .
\end{aligned}
$$

Proof. Using Equations (9), (10), (21) and (22), we can easily obtain the results (33) and (34). We omit the proof.

Theorem 5. Let $j \geq 0$, we have

$$
\begin{aligned}
& C_{j}(u, v)=F_{j}^{(c)}(u, v ; w)-w F_{j}^{(c)}(u+1, v ; w)+w F_{j}^{(c)}(u, v ; w), \\
& S_{j}(u, v)=F_{j}^{(s)}(u, v ; w)-w F_{j}^{(s)}(u+1, v ; w)+w F_{j}^{(s)}(u, v ; w) .
\end{aligned}
$$

Proof. From (21), we have

$$
\begin{gathered}
e^{u z} \cos v z=\frac{1-w\left(e^{z}-1\right)}{1-w\left(e^{z}-1\right)} e^{u z} \cos v z \\
=\frac{e^{u z} \cos v z}{1-w\left(e^{z}-1\right)}-\frac{w\left(e^{z}-1\right)}{1-w\left(e^{z}-1\right)} e^{u z} \cos v z .
\end{gathered}
$$

By using (9) and (21), we have

$$
\begin{gathered}
\sum_{j=0}^{\infty} C_{j}(u, v) \frac{z^{j}}{j !} \\
=\sum_{j=0}^{\infty}\left[F_{j}^{(c)}(u, v ; w)-w F_{j}^{(c)}(u+1, v ; w)+w F_{j}^{(c)}(u, v ; w)\right] \frac{z^{j}}{j !},
\end{gathered}
$$

which proves the desired result (35). The proof of (36) is similar.

Theorem 6. Let $j \geq 0$, we have

$$
\begin{aligned}
& w F_{j}^{(c)}(u+1, v ; w)=(1+w) F_{j}^{(c)}(u, v ; w)-C_{j}(u, v), \\
& w F_{j}^{(s)}(u+1, v ; w)=(1+w) F_{j}^{(s)}(u, v ; w)-S_{j}(u, v) .
\end{aligned}
$$

Proof. From (21), we see

$$
\begin{gathered}
\sum_{j=0}^{\infty}\left[F_{j}^{(c)}(u+1, v ; w)-F_{j}^{(c)}(u, v ; w)\right] \frac{z^{j}}{j !} \\
=\frac{e^{u z} \cos v z}{1-w\left(e^{z}-1\right)}\left(e^{z}-1\right) \\
=\frac{1}{w}\left[\frac{e^{u z} \cos v z}{1-w\left(e^{z}-1\right)}-e^{u z} \cos v z\right]
\end{gathered}
$$




$$
=\frac{1}{w} \sum_{j=0}^{\infty}\left[F_{j}^{(c)}(u, v ; w)-C_{j}(u, v)\right] \frac{z^{j}}{j !},
$$

which yields the obtained result (37). The proof of (38) is similar.

Theorem 7. For every $j \in \mathbb{N}$, we have

$$
\begin{gathered}
\frac{\partial}{\partial u} F_{j}^{(c)}(u, v ; w)=j F_{j-1}^{(c)}(u, v ; w), \\
\frac{\partial}{\partial v} F_{j}^{(c)}(u, v ; w)=-j F_{j-1}^{(s)}(u, v ; w),
\end{gathered}
$$

and

$$
\begin{gathered}
\frac{\partial}{\partial u} F_{j}^{(s)}(u, v ; w)=j F_{j-1}^{(s)}(u, v ; w), \\
\frac{\partial}{\partial v} F_{j}^{(s)}(u, v ; w)=j F_{j-1}^{(c)}(u, v ; w) .
\end{gathered}
$$

Proof. From (21), we have

$$
\begin{gathered}
\sum_{j=1}^{\infty} \frac{\partial}{\partial u} F_{j}^{(c)}(u, v ; w) \frac{z^{j}}{j !}=\frac{\partial}{\partial u} \frac{e^{u z} \cos v z}{1-w\left(e^{z}-1\right)}=\sum_{j=0}^{\infty} F_{j}^{(c)}(u, v ; w) \frac{z^{j+1}}{j !} \\
=\sum_{j=0}^{\infty} F_{j-1}^{(c)}(u, v ; w) \frac{z^{j}}{(j-1) !}=\sum_{j=1}^{\infty} j F_{j-1}^{(c)}(u, v ; w) \frac{z^{j}}{j !}
\end{gathered}
$$

proving (39). Other (40)-(42) can be similarly derived.

Theorem 8. Let $j \geq 0$, we have

$$
\begin{aligned}
& F_{j}^{(c)}(1+u, v ; w)=\sum_{r=0}^{j}\left(\begin{array}{c}
j \\
r
\end{array}\right) F_{j-r}^{(c)}(u, v ; w), \\
& F_{j}^{(s)}(1+u, v ; w)=\sum_{r=0}^{j}\left(\begin{array}{c}
j \\
r
\end{array}\right) F_{j-r}^{(s)}(u, v ; w) .
\end{aligned}
$$

Proof. Using the generating function (21), we have

$$
\begin{gathered}
\sum_{j=0}^{\infty} F_{j}^{(c)}(1+u, v ; w) \frac{z^{j}}{j !}-\sum_{j=0}^{\infty} F_{j}^{(c)}(u, v ; w) \frac{z^{j}}{j !} \\
=\left(\frac{1}{1-w\left(e^{z}-1\right)}\right)\left(e^{z}-1\right) e^{u z} \cos v z \\
=\sum_{j=0}^{\infty} F_{j}^{(c)}(u, v ; w) \frac{z^{j}}{j !}\left(\sum_{r=0}^{\infty} \frac{z^{r}}{r !}-1\right) \\
=\sum_{j=0}^{\infty} F_{j}^{(c)}(u, v ; w) \frac{z^{j}}{j !} \sum_{r=0}^{\infty} \frac{z^{r}}{r !}-\sum_{j=0}^{\infty} F_{j}^{(c)}(u, v ; w) \frac{z^{j}}{j !} \\
=\sum_{j=0}^{\infty} \sum_{r=0}^{j}\left(\begin{array}{c}
j \\
r
\end{array}\right) F_{j-r}^{(c)}(u, v ; w) \frac{z^{j}}{j !}-\sum_{j=0}^{\infty} F_{j}^{(c)}(u, v ; w) \frac{z^{j}}{j !},
\end{gathered}
$$


which gives the claimed result (43). The proof of (44) is similar.

Theorem 9. For $j \geq 0$ and $u_{1} \neq u_{2}$. Then

$$
\begin{gathered}
\sum_{k=0}^{j}\left(\begin{array}{c}
j \\
k
\end{array}\right) F_{j-k}^{(c)}\left(u_{1}, v_{1} ; w_{1}\right) F_{k}^{(c)}\left(u_{2}, v_{2} ; w_{2}\right) \\
=\frac{w_{2} F_{j}^{(c)}\left(u_{1}+u_{2}, v_{1}+v_{2} ; w_{2}\right)-w_{1} F_{j}^{(c)}\left(u_{1}+u_{2}, v_{1}+v_{2} ; w_{1}\right)}{w_{2}-w_{1}},
\end{gathered}
$$

and

$$
\begin{gathered}
\sum_{k=0}^{j}\left(\begin{array}{c}
j \\
k
\end{array}\right) F_{j-k}^{(s)}\left(u_{1}, v_{1} ; w_{1}\right) F_{k}^{(s)}\left(u_{2}, v_{2} ; w_{2}\right) \\
=\frac{w_{2} F_{j}^{(s)}\left(u_{1}+u_{2}, u_{1}+u_{2} ; w_{2}\right)-w_{1} F_{j}^{(s)}\left(u_{1}+u_{2}, v_{1}+v_{2} ; w_{1}\right)}{w_{2}-w_{1}} .
\end{gathered}
$$

Proof. Equation (21) can be written as

$$
\begin{gathered}
\sum_{j=0}^{\infty} \sum_{k=0}^{\infty} F_{j}^{(c)}\left(u_{1}, v_{1} ; w_{1}\right) F_{k}^{(c)}\left(u_{2}, v_{2} ; w_{2}\right) \frac{z^{j}}{j !} \frac{z^{k}}{k !} \\
=\frac{e^{u_{1} z} \cos \left(v_{1} z\right)}{1-w_{1}\left(e^{z}-1\right)} \frac{e^{u_{2} z} \cos \left(v_{2} z\right)}{1-w_{2}\left(e^{z}-1\right)} \\
=\frac{w_{2}}{w_{2}-w_{1}} \frac{e^{\left(u_{1}+u_{2}\right) z} \cos \left[\left(v_{1}+v_{2}\right) z\right]}{1-w_{1}\left(e^{z}-1\right)}-\frac{w_{1}}{w_{2}-w_{1}} \frac{e^{\left(u_{1}+u_{2}\right) z} \cos \left[\left(v_{1}+v_{2}\right) z\right]}{1-w_{2}\left(e^{z}-1\right)} \\
=\sum_{j=0}^{\infty}\left(\frac{w_{2} F_{j}^{(c)}\left(u_{1}+u_{2}, v_{1}+v_{2} ; w_{2}\right)-w_{1} F_{j}^{(c)}\left(u_{1}+u_{2}, v_{1}+v_{2} ; w_{1}\right)}{w_{2}-w_{1}}\right) \frac{z^{j}}{j !} .
\end{gathered}
$$

By equating the coefficients of $\frac{z^{j}}{j !}$ on both sides, we get (45). The proof of (46) is similar.

Theorem 10. For $j \geq 0$, we have

$$
(1+w) F_{j}^{(c)}(u, v ; w)=w \sum_{k=0}^{j}\left(\begin{array}{c}
j \\
k
\end{array}\right) F_{j-k}^{(c)}(u, v ; w)+C_{j}(u, v),
$$

and

$$
(1+w) F_{j}^{(s)}(u, v ; w)=w \sum_{k=0}^{j}\left(\begin{array}{c}
j \\
k
\end{array}\right) F_{j-k}^{(s)}(u, v ; w)+S_{j}(u, v)
$$

Proof. Consider the following identity

$$
\frac{1+w}{\left(1-w\left(e^{z}-1\right)\right) w e^{z}}=\frac{1}{1-w\left(e^{z}-1\right)}+\frac{1}{w e^{z}} .
$$

Using above identity by partial fraction, we find

$$
\frac{(1+w) e^{u z} \cos v z}{\left(1-w\left(e^{z}-1\right)\right) w e^{z}}=\frac{e^{u z} \cos v z}{1-w\left(e^{z}-1\right)}+\frac{e^{u z} \cos v z}{w e^{z}}
$$




$$
\begin{gathered}
(1+w) \sum_{j=0}^{\infty} F_{j}^{(c)}(u, v ; w) \frac{z^{j}}{j !} \\
=w \sum_{j=0}^{\infty} F_{j}^{(c)}(u, v ; w) \frac{z^{j}}{j !} \sum_{k=0}^{\infty} \frac{z^{k}}{k !}+\sum_{j=0}^{\infty} C_{j}(u, v) \frac{z^{j}}{j !},
\end{gathered}
$$

which implies the desired result (47). The proof of (48) is similar.

\section{Relationship between Appell-Type Polynomials}

In this section, we prove some relationships for parametric Fubini-type polynomials related to Bernoulli, Euler, and Genocchi polynomials and Stirling numbers of the second kind. We start the following theorem.

Theorem 11. For $j \geq 0$, we have

$$
\begin{aligned}
& F_{j}^{(c)}(u, v ; w) \\
& =\sum_{r=0}^{j+1}\left(\begin{array}{l}
j+1 \\
r
\end{array}\right)\left[\sum_{k=0}^{r}\left(\begin{array}{l}
r \\
k
\end{array}\right) B_{r-k}(u)-B_{r}(u)\right] \frac{F_{j+1-r}^{(c)}(0, v ; w)}{j+1},
\end{aligned}
$$

and

$$
\begin{aligned}
& F_{j}^{(s)}(u, v ; w) \\
& =\sum_{r=0}^{j+1}\left(\begin{array}{l}
j+1 \\
r
\end{array}\right)\left[\sum_{k=0}^{r}\left(\begin{array}{l}
r \\
k
\end{array}\right) B_{r-k}(u)-B_{r}(u)\right] \frac{F_{j+1-r}^{(s)}(0, v ; w)}{j+1} .
\end{aligned}
$$

Proof. From (1) and (21), we have

$$
\begin{aligned}
& \left(\frac{1}{1-w\left(e^{z}-1\right)}\right) e^{u z} \cos v z=\left(\frac{1}{1-w\left(e^{z}-1\right)}\right) \frac{z}{e^{z}-1} \frac{e^{z}-1}{z} e^{u z} \cos v z \\
& =\frac{1}{z} \sum_{j=0}^{\infty}\left(\sum_{k=0}^{s}\left(\begin{array}{c}
s \\
k
\end{array}\right) B_{s-k}(u)\right) \frac{z^{s}}{s !} \sum_{j=0}^{\infty} F_{j}^{(c)}(0, v ; w) \frac{z^{j}}{j !} \\
& -\frac{1}{z} \sum_{s=0}^{\infty} B_{s}(u) \frac{z^{s}}{s !} \sum_{j=0}^{\infty} F_{j}^{(c)}(0, v ; w) \frac{z^{j}}{j !} \\
& =\frac{1}{z} \sum_{j=0}^{\infty}\left[\sum_{r=0}^{j}\left(\begin{array}{c}
j \\
r
\end{array}\right) \sum_{k=0}^{r}\left(\begin{array}{c}
r \\
k
\end{array}\right) B_{r-k}(u)\right] F_{j-r}^{(c)}(0, v ; w) \frac{z^{j}}{j !} \\
& -\frac{1}{z} \sum_{j=0}^{\infty}\left[\sum_{r=0}^{j}\left(\begin{array}{c}
j \\
r
\end{array}\right) B_{r}(v)\right] F_{j-r}^{(c)}(0, v ; w) \frac{z^{j}}{j !}
\end{aligned}
$$

which gives the required result (49). The proof of (50) is similar.

Theorem 12. For $j \geq 0$, we have

$$
\begin{aligned}
& F_{j}^{(c)}(u, v ; w) \\
& =\sum_{r=0}^{j}\left(\begin{array}{c}
j \\
r
\end{array}\right)\left[\sum_{k=0}^{r}\left(\begin{array}{l}
r \\
k
\end{array}\right) E_{r-k}(u)+E_{r}(u)\right] \frac{F_{j-r}^{(c)}(0, v ; w)}{2},
\end{aligned}
$$


and

$$
\begin{aligned}
& F_{j}^{(s)}(u, v ; w) \\
& =\sum_{r=0}^{j}\left(\begin{array}{c}
j \\
r
\end{array}\right)\left[\sum_{k=0}^{r}\left(\begin{array}{l}
r \\
k
\end{array}\right) E_{r-k}(u)+E_{r}(u)\right] \frac{F_{j-r}^{(s)}(0, v ; w)}{2} .
\end{aligned}
$$

Proof. By using (2) and (21), we have

$$
\begin{aligned}
& \left(\frac{1}{1-w\left(e^{z}-1\right)}\right) e^{u z} \cos v z \\
& =\left(\frac{1}{1-w\left(e^{z}-1\right)}\right) \frac{2}{e^{z}+1} \frac{e^{z}+1}{2} e^{u z} \cos v z \\
& =\frac{1}{2}\left[\sum_{j=0}^{\infty}\left(\sum_{k=0}^{j}\left(\begin{array}{c}
j \\
k
\end{array}\right) E_{j-k}(u)\right) \frac{z^{j}}{j !}+\sum_{j=0}^{\infty} E_{j}(u) \frac{z^{j}}{j !}\right] \\
& \times \sum_{j=0}^{\infty} F_{j}^{(c)}(0, v ; w) \frac{z^{j}}{j !} \\
& =\frac{1}{2} \sum_{j=0}^{\infty}\left[\sum_{r=0}^{j}\left(\begin{array}{c}
j \\
r
\end{array}\right) \sum_{k=0}^{r}\left(\begin{array}{c}
r \\
k
\end{array}\right) E_{r-k}(u)+\sum_{r=0}^{j}\left(\begin{array}{c}
j \\
r
\end{array}\right) E_{r}(u)\right] \\
& \times F_{j-r}^{(c)}(0, v ; w) \frac{z^{j}}{j !}
\end{aligned}
$$

which arrives the desired result (51). The proof of (52) is similar.

Theorem 13. For $j \geq 0$, we have

$$
\begin{aligned}
& F_{j}^{(c)}(u, v ; w) \\
& =\frac{1}{2} \sum_{r=0}^{j+1}\left(\begin{array}{l}
j+1 \\
r
\end{array}\right)\left[\sum_{k=0}^{r}\left(\begin{array}{l}
r \\
k
\end{array}\right) G_{r-k}(u)+G_{r}(u)\right] \frac{F_{j+1-r}^{(c)}(0, v ; w)}{j+1},
\end{aligned}
$$

and

$$
\begin{aligned}
& F_{j}^{(s)}(u, v ; w) \\
& =\frac{1}{2} \sum_{r=0}^{j+1}\left(\begin{array}{l}
j+1 \\
r
\end{array}\right)\left[\sum_{k=0}^{r}\left(\begin{array}{l}
r \\
k
\end{array}\right) G_{r-k}(u)+G_{r}(u)\right] \frac{F_{j+1-r}^{(s)}(0, v ; w)}{j+1} .
\end{aligned}
$$

Proof. From (3) and (21), we have

$$
\begin{aligned}
& \left(\frac{1}{1-w\left(e^{z}-1\right)}\right) e^{u z} \cos v z \\
& =\left(\frac{1}{1-w\left(e^{z}-1\right)}\right) \frac{2 z}{e^{z}+1} \frac{e^{z}+1}{2 z} e^{u z} \cos v z \\
& =\frac{1}{2 z}\left[\sum_{j=0}^{\infty}\left(\sum_{k=0}^{j}\left(\begin{array}{c}
j \\
k
\end{array}\right) G_{j-k}(u)\right) \frac{z^{j}}{j !}+\sum_{j=0}^{\infty} G_{j}(u) \frac{z^{j}}{j !}\right] \\
& \times \sum_{j=0}^{\infty} F_{j}^{(c)}(0, v ; w) \frac{z^{j}}{j !},
\end{aligned}
$$

yields the asserted result (53). The proof of (54) is similar. 
Theorem 14. For $j \geq 0$, we have

$$
F_{j}^{(c)}(u, v ; w)=\sum_{r=0}^{j}\left(\begin{array}{l}
j \\
r
\end{array}\right) C_{j-r}(u, v) \sum_{k=0}^{r} z^{k} k ! S_{2}(r, k),
$$

and

$$
F_{j}^{(s)}(u, v ; w)=\sum_{r=0}^{j}\left(\begin{array}{c}
j \\
r
\end{array}\right) S_{j-r}(u, v) \sum_{k=0}^{r} z^{k} k ! S_{2}(r, k) .
$$

Proof. From (8) and (21), we have

$$
\begin{gathered}
\sum_{j=0}^{\infty} F_{j}^{(c)}(u, v ; w) \frac{z^{j}}{j !}=\frac{1}{1-w\left(e^{z}-1\right)} e^{u z} \cos v z \\
=e^{u z} \cos v z \sum_{k=0}^{\infty} z^{k}\left(e^{z}-1\right)^{k} \\
=e^{u z} \cos v z \sum_{k=0}^{\infty} z^{k} \sum_{r=k}^{\infty} k ! S_{2}(r, k) \frac{z^{r}}{r !} \\
=\sum_{j=0}^{\infty} C_{j}(u, v) \frac{z^{j}}{j !} \sum_{r=0}^{\infty} z^{k} \sum_{k=0}^{r} k ! S_{2}(r, k) \frac{z^{r}}{r !} .
\end{gathered}
$$

Replacing $j$ by $j-r$ in above equation, we get

$$
\begin{gathered}
\sum_{j=0}^{\infty} F_{j}^{(c)}(u, v ; w) \frac{z^{j}}{j !} \\
=\sum_{j=0}^{\infty}\left(\sum_{r=0}^{j}\left(\begin{array}{c}
j \\
r
\end{array}\right) C_{j-r}(u, v) \sum_{k=0}^{r} z^{k} k ! S_{2}(r, k)\right) \frac{z^{j}}{j !},
\end{gathered}
$$

which gives the asserted result (55). The proof of (56) is similar.

Theorem 15. Let $j \geq 0$, we have

$$
F_{j}^{(c)}(u+\alpha, v ; w)=\sum_{r=0}^{j}\left(\begin{array}{c}
j \\
r
\end{array}\right) C_{j-r} \sum_{k=0}^{r} w^{k} k ! S_{2}(r+\alpha, k+\alpha),
$$

and

$$
F_{j}^{(s)}(u+\alpha, v ; w)=\sum_{r=0}^{j}\left(\begin{array}{c}
j \\
r
\end{array}\right) S_{j-r} \sum_{k=0}^{r} w^{k} k ! S_{2}(r+\alpha, k+\alpha) .
$$

Proof. Replacing $u$ by $u+\alpha$ in (21) and using the result ([2], p. 250, Theorem 16), we have

$$
\begin{gathered}
\sum_{j=0}^{\infty} F_{j}^{(c)}(u+\alpha, v ; w) \frac{z^{j}}{j !}=\frac{1}{1-w\left(e^{z}-1\right)} e^{(u+\alpha) z} \cos v z \\
=e^{u z} \cos v z e^{\alpha z} \sum_{k=0}^{\infty} w^{k}\left(e^{z}-1\right)^{k} \\
=e^{u z} \cos v z e^{\alpha z} \sum_{k=0}^{\infty} w^{k} \sum_{r=k}^{\infty} k ! S_{2}(r, k) \frac{z^{r}}{r !}
\end{gathered}
$$




$$
=\sum_{j=0}^{\infty} C_{j}(u, v) \frac{z^{j}}{j !} \sum_{r=0}^{\infty} w^{k} \sum_{k=0}^{r} k ! S_{2}(r+\alpha, k+\alpha) \frac{z^{r}}{r !} .
$$

Replacing $j$ by $j-r$ in above equation, we get

$$
\begin{gathered}
\sum_{j=0}^{\infty} F_{j}^{(c)}(u+\alpha, v ; w) \frac{z^{j}}{j !} \\
=\sum_{j=0}^{\infty}\left(\sum_{r=0}^{j}\left(\begin{array}{c}
j \\
r
\end{array}\right) C_{j-r}(u, v) \sum_{k=0}^{r} w^{k} k ! S_{2}(r+\alpha, k+\alpha)\right) \frac{z^{j}}{j !} .
\end{gathered}
$$

Equating the coefficients of $z^{j}$ on both sides, we get (57). The proof of (58) is similar.

Theorem 16. Let $j \geq 0$, we have

$$
F_{j}^{(c)}(u, v ; w)=\sum_{r=0}^{\infty} \sum_{i=r}^{j}\left(\begin{array}{l}
j \\
i
\end{array}\right)(u)_{r} S_{2}(i, r) F_{j-i}^{(c)}(0, v ; w)
$$

and

$$
F_{j}^{(s)}(u, v ; w)=\sum_{r=0}^{\infty} \sum_{i=r}^{j}\left(\begin{array}{l}
j \\
i
\end{array}\right)(u)_{r} S_{2}(i, r) F_{j-i}^{(s)}(0, v ; w) .
$$

Proof. From (7) and (21), we find

$$
\begin{gathered}
\sum_{j=0}^{\infty} F_{j}^{(c)}(u, v ; w) \frac{z^{j}}{j !}=\frac{1}{1-w\left(e^{z}-1\right)}\left(\left(e^{z}-1\right)+1\right)^{u} \cos v z \\
=\left(\frac{\operatorname{Li}_{k}\left(1-e^{-z}\right)}{e^{z}-1}\right) \sum_{j=0}^{\infty}\left(\begin{array}{c}
u \\
r
\end{array}\right)\left(e^{z}-1\right)^{l} \\
=\sum_{r=0}^{\infty}(u)_{r} \frac{\left(e^{z}-1\right)^{r}}{r !}\left(\frac{1}{1-w\left(e^{z}-1\right)}\right) \cos v z \\
=\sum_{r=0}^{\infty}(u)_{r} \sum_{i=0}^{\infty} S_{2}(i, r) \frac{z^{i}}{i !} \sum_{j=0}^{\infty} F_{j}^{(c)}(0, v ; w) \frac{z^{j}}{j !} \\
=\sum_{j=0}^{\infty}\left(\sum_{r=0}^{\infty} \sum_{i=r}^{j}\left(\begin{array}{l}
j \\
i
\end{array}\right)(u)_{r} S_{2}(i, r) F_{j-i}^{(c)}(0, v ; w)\right) \frac{z^{j}}{j !},
\end{gathered}
$$

which provides the claimed result (59). The proof of (60) is similar.

Theorem 17. For $j \geq 0$, we have

$$
F_{j}^{(c)}(u+\alpha, v ; w)=\sum_{r=0}^{j} \sum_{k=0}^{j}\left(\begin{array}{l}
j \\
r
\end{array}\right) F_{j-r}^{(c)}(u, v ; w) S_{2}(r, k)(\alpha)_{k},
$$

and

$$
F_{j}^{(s)}(u+\alpha, v ; w)=\sum_{r=0}^{j} \sum_{k=0}^{j}\left(\begin{array}{l}
j \\
r
\end{array}\right) F_{j-r}^{(s)}(u, v ; w) S_{2}(r, k)(\alpha)_{k} .
$$


Proof. From (8) and (21), we see

$$
\begin{gathered}
\sum_{j=0}^{\infty} F_{j}^{(c)}(u, v ; w) \frac{z^{j}}{j !}=\frac{1}{1-w\left(e^{z}-1\right)} e^{u z} \cos v z e^{\alpha z} \\
=\sum_{j=0}^{\infty} F_{j}^{(c)}(u, v ; w) \frac{z^{j}}{j !} \sum_{r=0}^{\infty} \frac{\alpha^{r} z^{r}}{r !} \\
=\sum_{j=0}^{\infty} \sum_{r=0}^{j}\left(\begin{array}{l}
j \\
r
\end{array}\right) F_{j-r}^{(c)}(u, v ; w) \alpha^{r} \frac{z^{r}}{r !} \\
=\sum_{j=0}^{\infty} \sum_{r=0}^{j}\left(\begin{array}{l}
j \\
r
\end{array}\right) F_{j-r}^{(c)}(u, v ; w) \frac{z^{j}}{j !} \sum_{k=0}^{r} S_{2}(r, k)(\alpha)_{k} \\
=\sum_{j=0}^{\infty} \sum_{r=0}^{j} \sum_{k=0}^{j}\left(\begin{array}{l}
j \\
r
\end{array}\right) F_{j-r}^{(c)}(u, v ; w) S_{2}(r, k)(\alpha)_{k} \frac{z_{j}^{j}}{j !} .
\end{gathered}
$$

Equating the coefficients $z^{j}$ on both sides, we get (61). The proof of (62) is similar.

Theorem 18. For $j \geq 0$, we have

$$
F_{j}^{(c)}(u, v ; w)=\sum_{k=0}^{\infty} \sum_{l=k}^{j}\left(\begin{array}{l}
j \\
l
\end{array}\right) F_{j-l}^{(c)}(-k, v ; w) S_{2}(l, k)(u)^{(k)},
$$

and

$$
F_{j}^{(s)}(u, v ; w)=\sum_{k=0}^{\infty} \sum_{l=k}^{j}\left(\begin{array}{l}
j \\
l
\end{array}\right) F_{j-l}^{(s)}(-k, v ; w) S_{2}(l, k)(u)^{(k)}
$$

Proof. From Equations (8) and (21), we determine

$$
\begin{gathered}
\sum_{j=0}^{\infty} F_{j}^{(c)}(u, v ; w) \frac{z^{j}}{j !}=\frac{\cos v z}{1-w\left(e^{z}-1\right)} e^{u z} \\
=\frac{\cos v z}{1-w\left(e^{z}-1\right)}\left(e^{-z}\right)^{-u}=\frac{\cos v z}{1-w\left(e^{z}-1\right)} \sum_{k=0}^{\infty}\left(\begin{array}{c}
u+k-1 \\
k
\end{array}\right)\left(1-e^{-z}\right)^{-k} \\
=\frac{\cos v z}{1-w\left(e^{z}-1\right)} \sum_{k=0}^{\infty}(u)^{(k)} \frac{\left(e^{z}-1\right)^{k}}{k !} e^{-k z} \\
=\sum_{k=0}^{\infty}(u)^{(k)} \sum_{j=0}^{\infty} F_{j}^{(c)}(-k, v ; w) \frac{z^{j}}{j !} \sum_{l=0}^{\infty} S_{2}(l, k) \frac{z^{l}}{l !} \\
=\sum_{k=0}^{\infty}(u)^{(k)} \sum_{j=0}^{\infty}\left(\sum_{l=0}^{j}\left(\begin{array}{l}
j \\
l
\end{array}\right) F_{j-l}^{(c)}(-k, v ; w) S_{2}(l, k)\right) \frac{z^{j}}{j !} .
\end{gathered}
$$

Comparing the coefficients $z^{j}$ on both sides, we get (63). The proof of (64) is similar.

\section{Conclusions}

In our present investigation, we have introduced and studied systematically two parametric families of Fubini polynomials $F_{j}^{(c)}(u, v ; w)$ and $F_{j}^{(s)}(u, v ; w)$, which are defined using two specific generating functions. We have derived several fundamental properties of these parametric kinds of Fubini polynomials and such other polynomials as the parametric kind Bernoulli, Euler, and Genocchi 
polynomials. Lastly, we show that complex cosine-Fubini polynomials and complex sine-Fubini polynomials can be bespoke in terms of first- and second-form Stirling numbers.

Author Contributions: Conceptualization, S.K.S.; formal analysis, W.A.K.; investigation, S.K.S., W.A.K. and C.S.R.; project administration, W.A.K.; supervision, C.S.R.; funding acquisition, S.K.S; writing-original draft, S.K.S. and W.A.K. All authors contributed equally to the manuscript and all authors have read and agreed to the published version of the manuscript.

Funding: Sunil Kumar Sharma would like to thank Deanship of Scientific Research at Majmaah University for supporting this work under the Project No. R-1441-113.

Acknowledgments: Sunil Kumar Sharma would like to acknowledge Mohammed Alshehri, College of Computer and Information Sciences, Majmaah University for his encouragement. The authors would like to thank the referees for their valuable comments and suggestions.

Conflicts of Interest: The authors declare no conflict of interest.

\section{References}

1. Boyadzhiev, K.N. A series transformation formula and related polynomials. Int. J. Math. Math. Sci. 2005, 23, 3849-3866. [CrossRef]

2. Broder, A.Z. The $r$-Stirling numbers. Discret. Math. 1984, 49, 241-259. [CrossRef]

3. Carlitz, L. Eulerian numbers and polynomials. Math. Mag. 1959, 32, 164-171. [CrossRef]

4. Dil, A.; Kurt, V. Investing geometric and exponential polynomials with Euler-Seidel matrices. J. Integer Seq. 2011, 14, 1-12.

5. Guohui, C.; Li, C. Some Identities Involving the Fubini Polynomials and Euler Polynomials. Mathematics 2018, 6, 300. [CrossRef]

6. Jamei, M.M.; Beyki, M.R.; Koepf, W. A new type of Euler polynomials and numbers. Mediterr. J. Math. 2018, 15, 138. [CrossRef]

7. Jamei, M.M.; Beyki, M.R.; Koepf, W. An extension of the Euler-Maclaurin quadrature formula using a parametric type of Bernoulli polynomials. Bull. Sci. Math. 2019, 156, 102798. [CrossRef]

8. Jamei, M.M.; Beyki, M.R.; Omey, E. On a parametric kind of Genocchi polynomials. J. Inequal. Spec. Funct 2018, 9, 68-81.

9. Jamei, M.M.; Beyki, M.R.; Koepf, W. Symbolic computation of some power trigonometric series. J. Symb. Comput. 2017, 80, 273-284. [CrossRef]

10. Khan, W.A. A new class of degenerate Frobenius-Euler-Hermite poynomials. Adv. Stud. Contemp. Math. (Kyungshang) 2018, 4, 567-576.

11. Kim, T.; Ryoo, C.S. Some identities for Euler and Bernoulli polynomials and their zeros. Axioms 2018, 7, 56. [CrossRef]

12. Kim, T.; Lee, B. Some identities of the Frobenius-Euler polynomials. Abst. Appl. Anal. 2008, $2009,639439$. [CrossRef]

13. Kim, D.S.; Kim, T.; Lee, H. A note on degenerate Euler and Bernoulli polynomials of complex variable. Symmetry 2019, 11, 1168. [CrossRef]

14. KIm, T.; KIm, D.S. A Note on central Bell Number and polynomials. Russ. J. Math. Phys. 2020, $27,79-81$. [CrossRef]

15. Kim, T.; Khan, W.A.; Sharma, S.K.; Ghayasuddin, M. A note on parametric kinds of the degenerate poly-Bernoulli and poly-Genocchi polynomials. Symmetry 2020, 12, 614. [CrossRef]

16. Kim, T.; Kim, D.S.; Dolgy, D.V.; Kwon, J. Some identities on generalized degenerate Genocchi and Euler nubers. Informatica 2020, 31, 42-51.

17. Kargin, L. Some formulae for products of Fubini polynomials with applications. arXiv 2016, arXiv:1701.01023v1[math.CA].

18. Lu, D.Q.; Srivastava, H.M. Some series identities involving the generalized Apostol type and related polynomials. Comput. Math. Appl. 2011, 62, 3591-3602. [CrossRef]

19. Luo, Q.M.; Srivastava, H.M. Some generalizations of the Apostol-Genocchi polynomials and the Stirling numbers of the second kind. Appl. Math. Comput. 2011, 217, 5702-5728. [CrossRef]

20. Qi, F. On Generalized Fubini Polynomials. HAL Id: Hal-01853686. 2018. Available online: http://hal. archives-ouvertes.fr/hal-0185368v1 (accessed on 31 March 2020). 
21. Ryoo, C.S.; Khan, W.A. On two bivariate kinds of poly-Bernoulli and poly-Genocchi polynomials. Mathematics 2020, 8, 417. [CrossRef]

22. Sharma, S.K.; Khan, W.A.; Ryoo, C.S. A parametric kind of the degenerate Fubini numbers and polynomials. Mathematics 2020, 8, 405. [CrossRef]

23. Srivastava, H.M.; Kizilates, C. A parametric kind of the Fubini-type polynomials. Rev. Real Acad. Sci. Exact. Fis. Nat. Ser. A Math. 2019, 113, 3253-3267. [CrossRef]

24. Srivastava, H.M.; Ricci, P.E.; Natalini, P. A family of complex Appell polynomial sets. Rev. Real Acad. Sci. Exact. Fis. Nat. Ser. A Math. 2019, 113, 2359-2371. [CrossRef]

25. Srivastava, H.M.; Jamei, M.M.; Beyki, M.R. Some new generalizations and applications of the Apostol-Bernoulli, Apostol-Euler and Apostol-Genocchi polynomials. Rocky Mt. J. Math. 2019, 49, 681-697. [CrossRef]

26. Tanny, S.M. On some numbers related to Bell numbers. Can. Math. Bull. 1974, 17, 733-738. [CrossRef]

2020 by the authors. Licensee MDPI, Basel, Switzerland. This article is an open access article distributed under the terms and conditions of the Creative Commons Attribution (CC BY) license (http:/ / creativecommons.org/licenses/by/4.0/). 